\title{
Conf-950550--4
}

\section{AIAA-95-1558 \\ Computational Fluid Dynamic Studies of a Solid and Ribbon 12-Gore Parachute Canopy in Subsonic and Supersonic Flow}

James M. Nelsen

Sandia National Laboratories

Albuquerque, NM 87185

The Goverment reserves for itself and others acting on its behalf a royalty free, nonexclusive, irrevocable, world-wide iicense for govermental purposes to publish, distribute, translate, duplicate, exhibit, and perform any such data copyrighted by the contractor.

\section{AIAA 13th Aerodynamic Decelerator Systems Technology Conference}

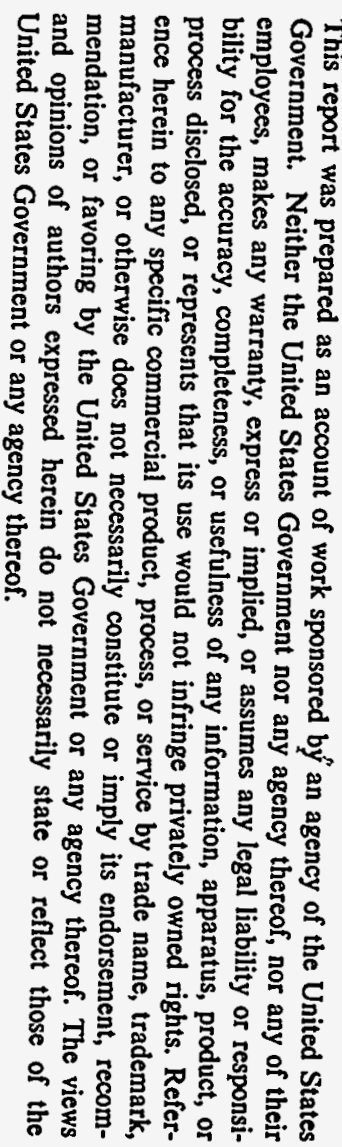

May 15-18, 1995 / Clearwater Beach, FL

For permission to copy or republish, contact the American Institute of Aeronautics and Astronautics 370 L'Enfant Promenade, S. W., Washington, D. C. 20024

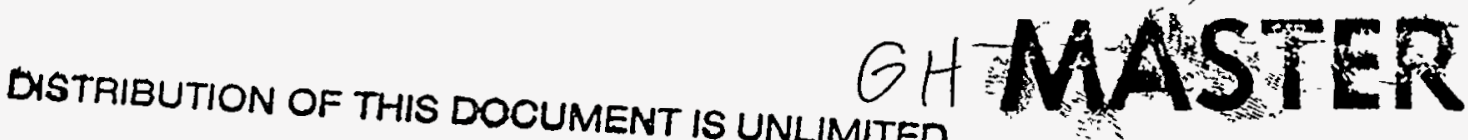




\section{DISCLAIMER}

Portions of this document may be illegible in electronic image products. Images are produced from the best available original document. 


\title{
Computational Fluid Dynamic Studies of a Solid and Ribbon 12-Gore Parachute Canopy in Subsonic and Supersonic Flow
}

\author{
James M. Nelsen ${ }^{\dagger}$ \\ Engineering Sciences Center \\ Sandia National Laboratories \\ Albuquerque, NM 87185
}

\begin{abstract}
Computational fluid dynamic studies of 3-D, fixed geometry, gore-shaped parachute canopies are presented. Both solid and ribbon canopies with a $10 \%$ vent diameter are investigated. The flowfields analyzed are laminar and compressible, broaching both the subsonic and supersonic regimes. Results presented include characterization of the local and global flowfields and the internal and external canopy surface pressure distributions. The canopy surface pressure distributions may be utilized in subsequent structural analyses to assess the integrity of the parachute canopy fabric components.
\end{abstract}

\section{Introduction}

Optimization of textile materials used in the manufacture of high performance fabric structures remains an important, yet largely unresolved issue. Application of empirical data combined with approximate analysis $[1,2,3,4]$ constitutes the traditional approach to fabric component selection in the design process. This approach, however, generally results in a nominal design which incorporates a relatively large factor-of-safety to accommodate the transient, dynamic loading. Further, instrumented testing is required to address such issues as the global, integrated effect of a local material refinement and to identify and assess potential design weaknesses in the structure.

Computational structural analyses [5] have achieved sufficient sophistication to address the deficiencies cited above. Necessary input data for an analysis code [6] includes specification of the structure's design and component properties, the initial geometry, boundary restraints and the applied transient pressure field acting on the structure. Although the induced stress state in the fabric structure is inherently driven by the applied loading, resolution of the pressure field remains the weak link in the structural analysis modeling. Unquestionably, the paucity of experimental results [7] can be attributed to the dif-

† Senior Member of Technical Staff, Fluid/Structure Interactions Department, Senior Member of AIAA.

$\ddagger$ This work performed at Sandia National Laboratorics supported by the U.S. Department of Energy under contract number DEAC04-94AL85000.

This paper is declared a work of the U.S. Government and is not subject to copyright protection in the United States. ficulty in obtaining quality pressure measurements on a flexible structure which is both unstationary and deforming in the resulting unsteady flowfield.

The current study presents results of computational fluid dynamic (CFD) analyses of fixed 3-D parachute canopy geometries (i.e., fully inflated solid and ribbon) in subsonic and supersonic flowfields. In addition to examining the flowfield characteristics of these bluff body analyses, computed pressure distributions on the internal and external canopy geometries are presented.

\section{The Computational Models}

\section{Canopy Solid Models}

A computational model was developed which attempted to replicate the canopy portion of a 12-gore parachute with a $10 \%$ vent diameter. The model, which was derived mathematically, superimposes sinusoidal gore bulges (both latitudinally and longitudinally) on a spherical base, achieving a maximum gore bulge of approximately $20 \%$ of the base spherical radius. The model also incorporates flat sections (very low curvature in the latitudinal direction) separating the gore structures, which simulate the stiffer radials in a parachute. The model also extends beyond the longitudinal truncation of a hemisphere, representing the influence of the suspension lines which transition from the skirt of a parachute canopy to their confluence point.

Two distinct versions of this gore parachute geometry are analyzed: a solid model, which is shown in Figure 1 and a 13-ribbon model, which is shown in Figure 2. Each of the ribbons in the second model is of identical width and the ratio of ribbon width to gap is 2:1 (this construction is arbitrary and may not reflect acceptable design practices). To minimize edge effects during the fluid dynamic analyses, both canopy models were constructed with zero thickness. The solid models were generated using the commercially available code PATRAN [8].

\section{Canopy Surface Mesh Models}

Figure 3 displays the surface mesh utilized in the current analysis for the solid canopy model. This surface mesh is comprised of 29,126 unstructured, triangular cells. The ribbon canopy surface mesh, shown in Figure 4, is comprised of 32,522 (substantially) structured, triangular 


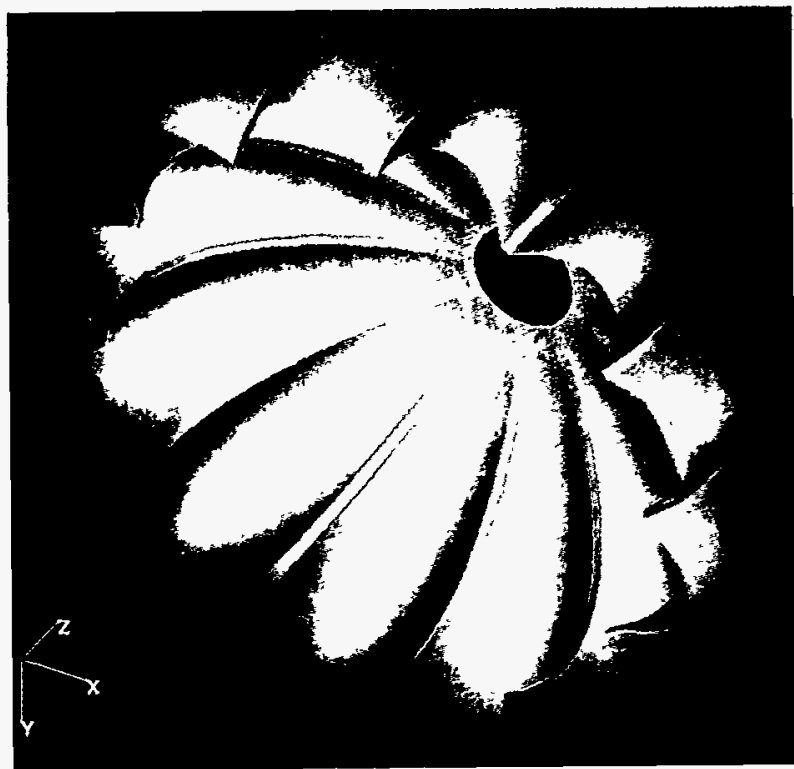

Figure 1. Solid canopy parachute model.

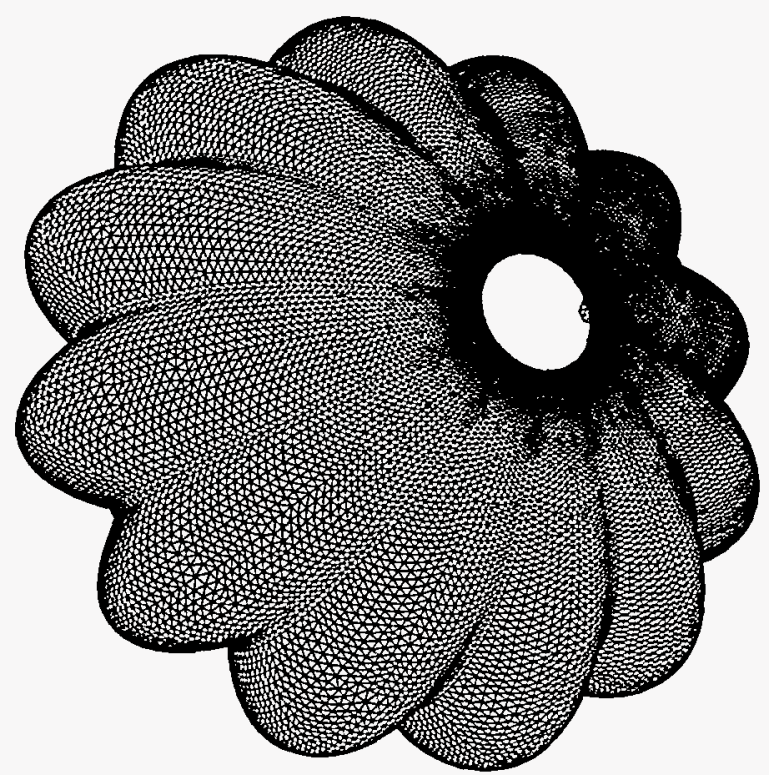

Figure 3. Solid canopy surface mesh.

cells. Uniformity of meshing between each gore structure was achieved by meshing an individual gore and revolving the surface mesh to the adjacent gores.

Specifically referencing the ribbon canopy surface mesh, a minimum of two fluid cells across the gap was essential to model the flow through the gaps in a viscous flowfield. This requirement, further constrained by the desire to preserve a reasonable cell skewness, dictated a minimum of four surface cells across the ribbon width.

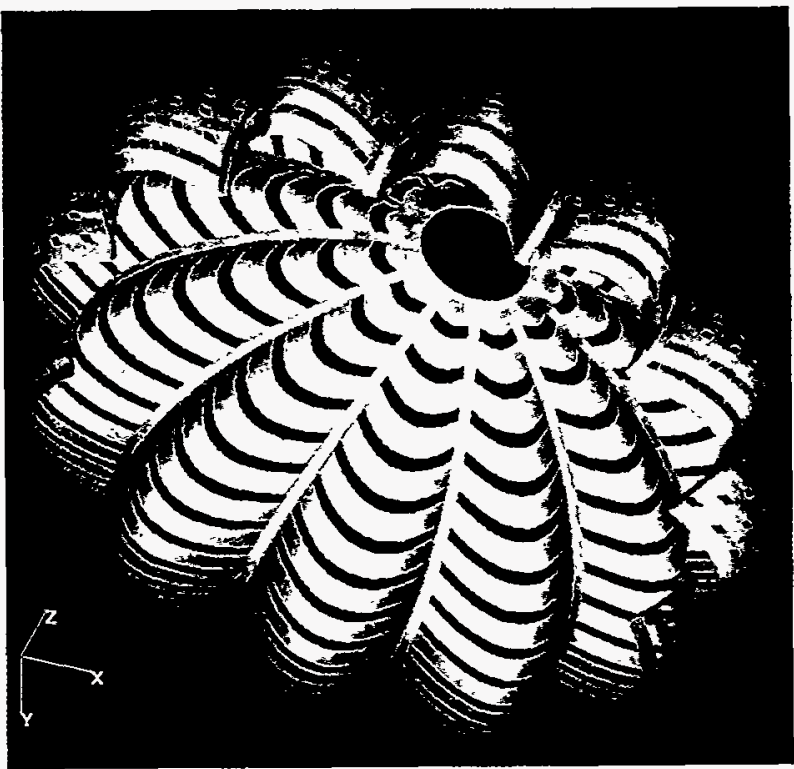

Figure 2. Ribbon canopy parachute model.

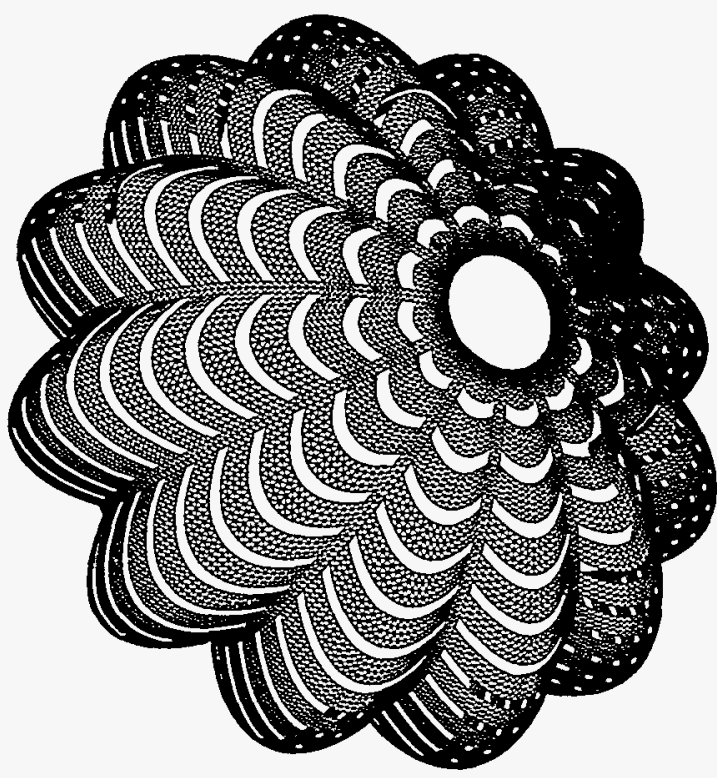

Figure 4. Ribbon canopy surface mesh.

Two cells were also implemented across the flat-section "radial" width. The surface mesh models were also generated using PATRAN.

The Flow Domain Model

The flow domain, which was modeled as a cylinder, is shown in Figure 5. The outer dimensions were sized such that the imposed far-field boundary conditions would not interfere with or compromise the flowfield characteristics generated by the bluff body canopy models. Relative 


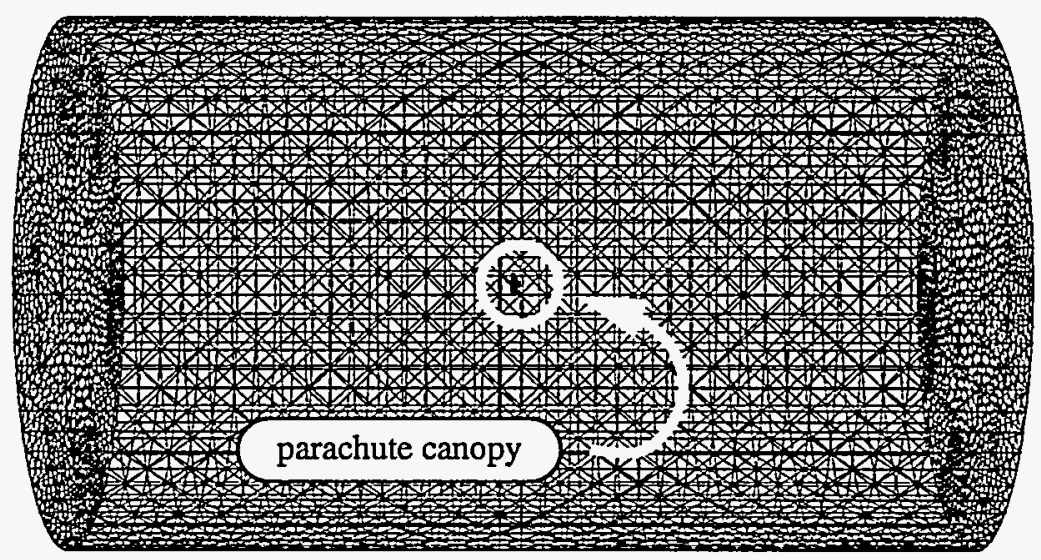

Figure 5. Flow domain model with canopy identified.

to the maximum canopy diameter (i.e., referred to as the inflated diameter in parachute terminology), the flowfield inlet is 20 diameters upstream, the outlet is 21 diameters downstream and the cylindrical sidewall is $12 \mathrm{di}$ ameters distant from the canopy centerline. This flow domain was utilized for both the subsonic and supersonic cases studied. Figure 5 graphically identifies the relative position of the canopy model within the flow domain. The cylindrical far-field model and surface mesh were also generated with PATRAN.

\section{The Fluid Dynamics Simulation}

\section{Background}

Two separate laminar, compressible flowfield analyses were conducted on the two canopy geometries. The first considered a high subsonic case, with the freestream Mach number registering 0.75 . The second established the freestream Mach number at the low supersonic case of 1.5 .

The commercially available code Rampant [9] was used to conduct the computational fluid dynamics simulation. Rampant utilizes an unstructured grid, finite volume solver, with capabilities to model both Euler and NavierStokes flows, including compressible or incompressible, and laminar or turbulent options.

The time-accurate solver, although tedious, was used in these analyses, as the non-time-accurate algorithm demonstrated undesirable convergence attributes for these bluff body simulations. However, due to the implementation of the applied initial condition (i.e., instantaneously positioning the canopy into a uniform, freestream fluid domain), the analyses cannot be considered realistic time-accurate simulations.

To satisfy the Courant-Friedrichs-Lewy (CFL) constraint, the time-steps utilized were generally less than a microsecond. The total simulation time was limited to 30 milliseconds, which required approximately 100 days of Sparc 10, Model 61 CPU time. The solutions achieved a semi-converged, quasi-steady solution (local to the parachute canopy) during this simulation time.

\section{Flow Domain Discretization}

Rampant requires a polyhedral volume mesh to model the fluid domain. The companion 3-D meshing code for Rampant, TGrid [10], was utilized to generate the tetrahedral volume mesh connecting the canopy and far-field domain surface meshes. While the same volume mesh was used for the subsonic and supersonic cases, the two canopies generated different volume meshes due to their inherent geometric and surface mesh differences. The solid canopy flow domain contained 535,414 tetrahedral cells, while the ribbon canopy flow domain contained 563,968 cells. The total number of cells, which is a function of the tetrahedral cell refinement scheme within TGrid, continued until the maximum fluid cell skewness achieved a level considered acceptable within the Rampant solver.

It should be noted that Rampant also has the capability to adapt the fluid mesh during the simulation according to gradients of any of the fluid properties. This is a useful feature in capturing certain flowfield characteristics such as shocks, boundary layers, wake regions, etc. However, the adaption capability was not utilized in these analyses since the refinement procedure often produces highly skewed sliver cells, which may adversely impact the flow calculations.

\section{Boundary Conditions}

Depending on the flowfield physics being modeled, Rampant has several types of boundary conditions (BC's) which could be applied, including mass-flow-inlets, pressure-inlets and outlets or pressure-far-fields, subsonic-inlets and both solid and porous walls. Based on previous experience with comparable simulations, the far-field static pressure boundary condition was applied to the flow domain boundaries, while the imporous (solid) wall boundary condition was applied to the two canopies for all cases considered in this study. Application 
of this far-field $\mathrm{BC}$ required specification of the freestream static pressure, Mach number, fluid density and the directional components of the velocity vector. For all cases considered in this study, the freestream velocity vector is aligned to achieve a zero canopy angle-of-attack. Finally, the thermal BC implemented at the canopy is an adiabatic wall condition.

\section{Freestream Fluid Properties}

The freestream fluid properties used in these analyses were designated as air at sea level from the U.S. Standard Atmosphere [11]. These static properties remain fixed throughout the duration of the analyses. For future reference, the speed of sound at these conditions is $340 \mathrm{~m} / \mathrm{s}$.

\section{Results of the CFD Analyses}

\section{Introductory Remarks}

Prior to presenting the results of the CFD analyses, some general observations are discussed which are relevant to all the cases investigated. Figure 6 exhibits a centerline plane-slice of the scalar momentum magnitude for the solid canopy in the Mach 0.75 flowfield. It is clearly evident that the far-field boundary condition has negligible influence on the generated flowfield characteristics from the bluff body canopy. Also, as depicted in Figure 6, the analysis predicts a relatively small dimensional magnitude (momentum defect) for the wake region, which is counterintuitive. The principle cause of this wake anomaly is the acknowledgement of insufficient simulation time, thereby not permitting far-field wake development. Also contributing to this issue are: the non-optimal mesh definition in the wake region, the dissipative effect of numerical viscosity and the blending of results due to the implementation of the discrete color spectrum.

\section{Results of the Subsonic, Solid Canopy Analysis}

The results of the CFD analysis for the solid canopy in the Mach 0.75 flowfield are presented in Figures $7-11$. Figures 7 and 8, which are centerline plane-slices of the velocity magnitude and static pressure respectively, identify the expected flowfield features: the substantially stagnant flow inside the canopy, the pressure disturbance which propagates upstream of the canopy, the low pressure wake region and the acceleration of flow around the skirt and through the vent. Additional details include: the weak supersonic expansion through the vent opening, which functions as a nozzle; the development of a local supersonic region external to and enveloping the near wake region; the development of counter-rotating toroidal vortices depicted in Figure 11: a small one near the vent rotating clockwise and the primary one between the vent and the skirt, which accelerates near the canopy due to the favorable pressure gradient; and the formation of two stagnation arcs between the vent and the skirt, which are consistent with the development of the counter-rotating toroidal vortices.

The internal and external canopy surface pressures are shown in Figures 9 and 10, respectively. The initial ob-

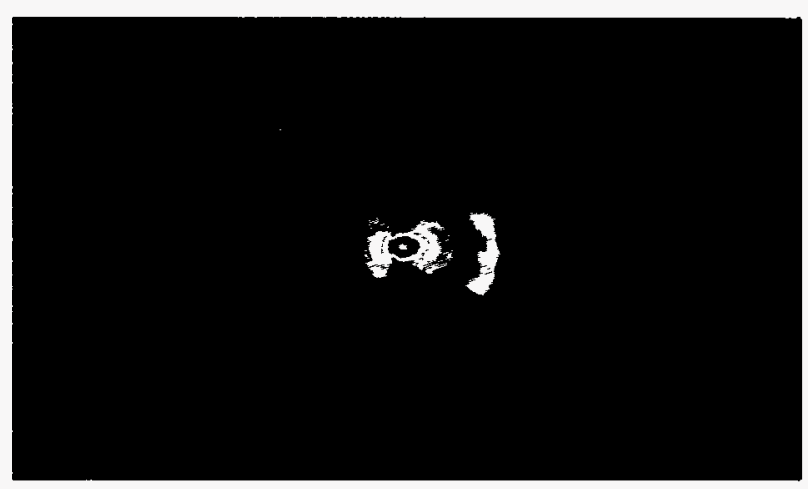

Figure 6. Centerline plane-slice of momentum magnitude.

servation is that the surface pressures are essentially axisymmetric. Also, the internal canopy pressure is substantially uniform, with less than a $10 \%$ gradient between the lower pressure skirt and the higher pressure vent region (excluding the skirt and vent band). The external canopy pressure distribution experiences significantly larger gradients and is non-monotonic, varying from the $90 \mathrm{kPa}$ levels at the maximum inflated diameter just forward of the skirt band (i.e., the location of the primary toroidal vortex stagnation arc) to the $40 \mathrm{kPa}$ level along the gore longitudinal mid-arc location, increasing again to approximately $73 \mathrm{kPa}$ in a region surrounding the vent opening.

One final observation concerning the internal canopy pressure is noteworthy. The maximum canopy surface pressure is observed to be $155 \mathrm{kPa}$, which exceeds the maximum physically realistic isentropic stagnation pressure $(-147 \mathrm{kPa})$ by approximately $5 \%$. This pressure anomaly is a result of the "impulsively started" initial condition which was applied and further emphasizes the "semi-converged solution" statement expressed previously, since the "added mass term" transient dynamics have not expired.

Canopy differential surface pressure results are not directly attainable with the current post-processing capabilities. Therefore, remarks concerning the differential values are reported as an approximate composite of the internal and external surface pressure values. The canopy differential surface pressure ranges from $80 \mathrm{kPa}$ at the vent region, to a maximum value of $105 \mathrm{kPa}$ at the longitudinal mid-gore region, decreasing to approximately 55 $\mathrm{kPa}$ at the maximum inflated diameter and 15 to $25 \mathrm{kPa}$ at the skirt.

\section{Results of the Subsonic, Ribbon Canopy Analysis}

The results of the CFD analysis for the ribbon canopy in the Mach 0.75 flowfield are presented in Figures 12-16. Considered on a global scale, the flowfield results are not significantly different than the solid canopy case. Generally, the observations are intuitive: the ribbon canopy, by virtue of it's geometric porosity, disturbs the flowfield less and the flow is not quite as stagnant in the canopy. 


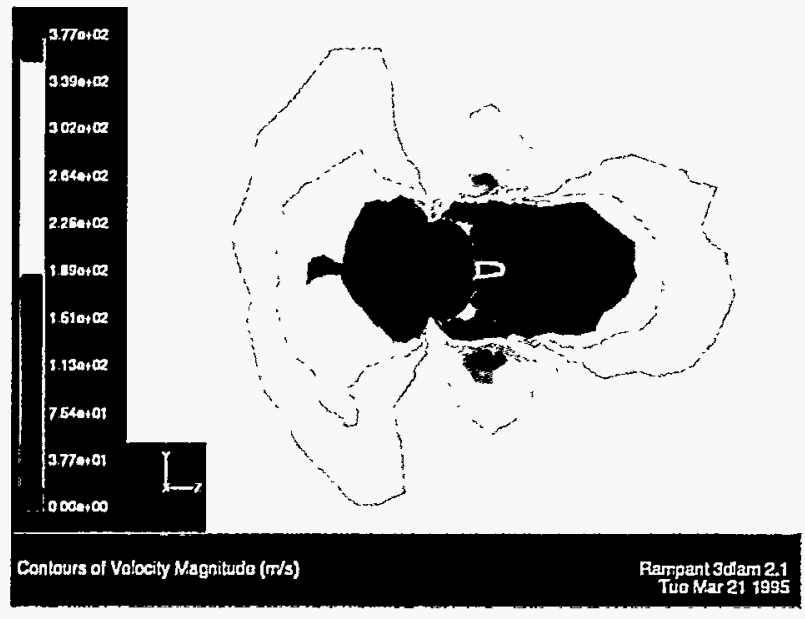

Figure 7. Centerline plane-slice of velocity magnitude. ( Solid canopy, Mach 0.75 flowfield.)

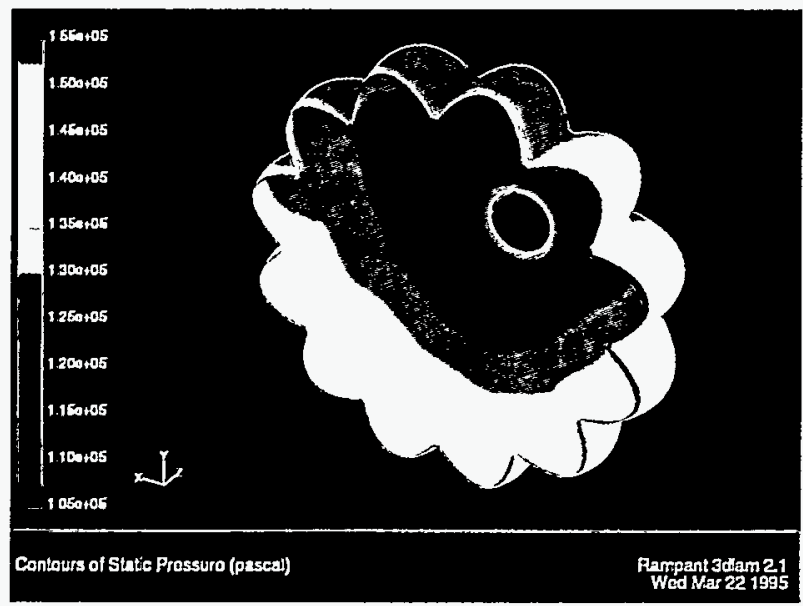

Figure 9. Canopy internal surface pressure distribution. ( Solid canopy, Mach 0.75 flowfield.)

Also, the toroidal vortices, if they are present, are certainly diminished and translated further back into the wake region. As shown in Figure 16, there is a vortex formation on the aft side of the ribbon, which is consistent with the flow through the gaps.

Neglecting ribbon edge effects, the internal canopy pressure distribution is monotonic and substantially uniform, displaying a $10 \%$ gradient between the skirt and the vent. The external canopy pressure distribution is essentially uniform along the latitudinal mid-gore region, with the exception of the significant increase in pressure at the skirt region. However, the distribution is non-axisymmetric, exhibiting significantly lower pressures in the radial troughs (especially in the longitudinal mid-gore region).

The canopy differential surface pressure results range from $80 \mathrm{kPa}$ at the vent region, to a maximum of $100 \mathrm{kPa}$

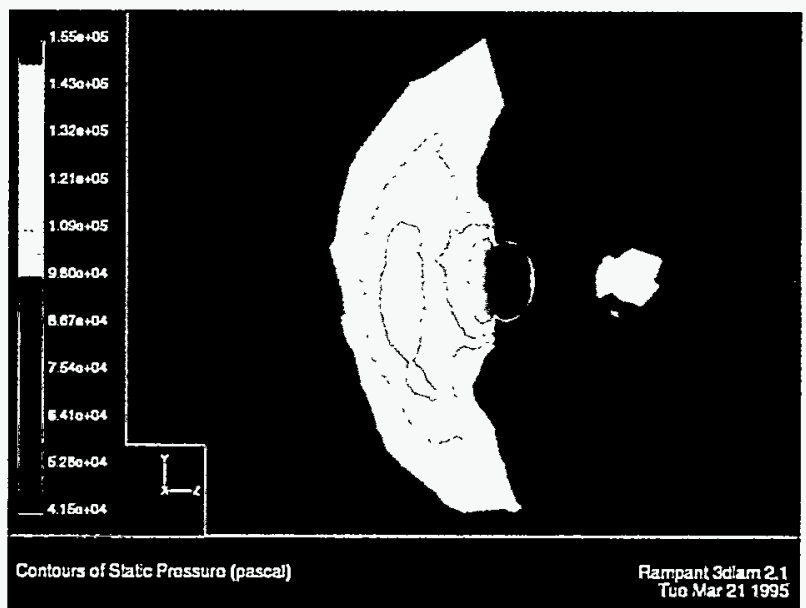

Figure 8. Centerline plane-slice of static pressure. ( Solid canopy, Mach 0.75 flowfield.)

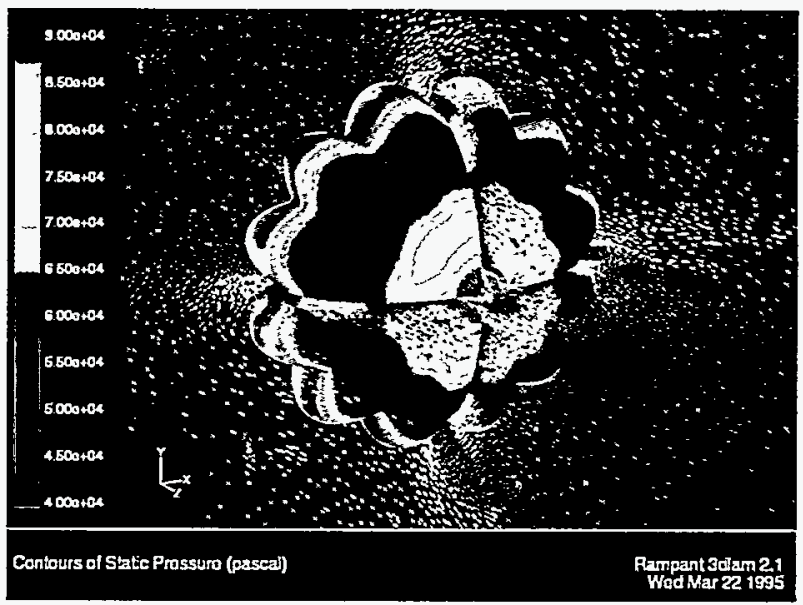

Figure 10. Canopy external surface pressure distribution. ( Solid canopy, Mach 0.75 flowfield.)

at the longitudinal mid-gore region in the radial troughs, decreasing to approximately $30 \mathrm{kPa}$ in the skirt region. The edges of each ribbon, however, sustain negligible pressure difference. Structurally, this implies that a flexible ribbon would deform to accommodate longitudinal curvature and may introduce flutter problems.

\section{Results of the Supersonic. Solid Canopy Analysis}

The results of the CFD analysis for the solid canopy in the Mach 1.5 flowfield are presented in Figures 17-21. The flowfield features are characteristically supersonic: the upstream disturbance develops a classic bluff body bow shock, though not sharply defined (due to the absence of grid adaption) and the supersonic expansion behind the bow shock evolves into a Mach cone. Analogous to the subsonic, solid canopy case, the flow inside the canopy is substantially stagnant, while the flow through the "nozzle" vent accelerates beyond Mach 2. 


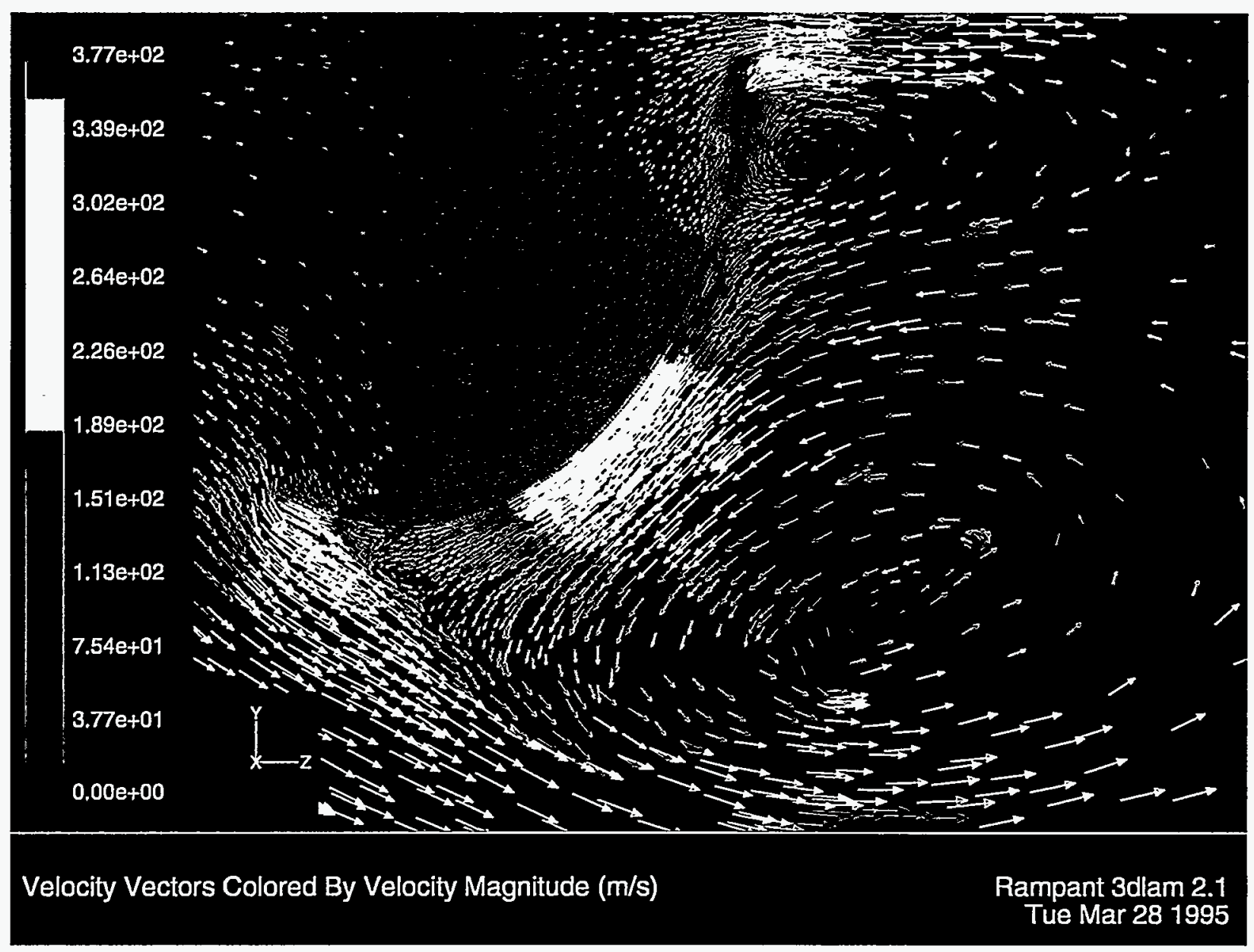

Figure 11. Centerline plane-slice of velocity vectors exhibiting the toroidal vortices.

( Solid canopy, Mach 0.75 flowfield.)

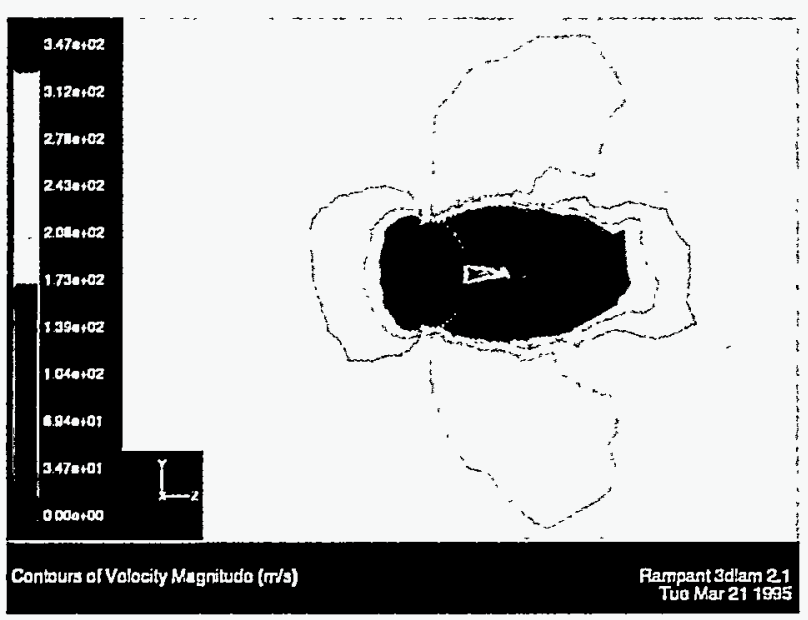

Figure 12. Centerline plane-slice of velocity magnitude. ( Ribbon canopy, Mach 0.75 flowfield.)

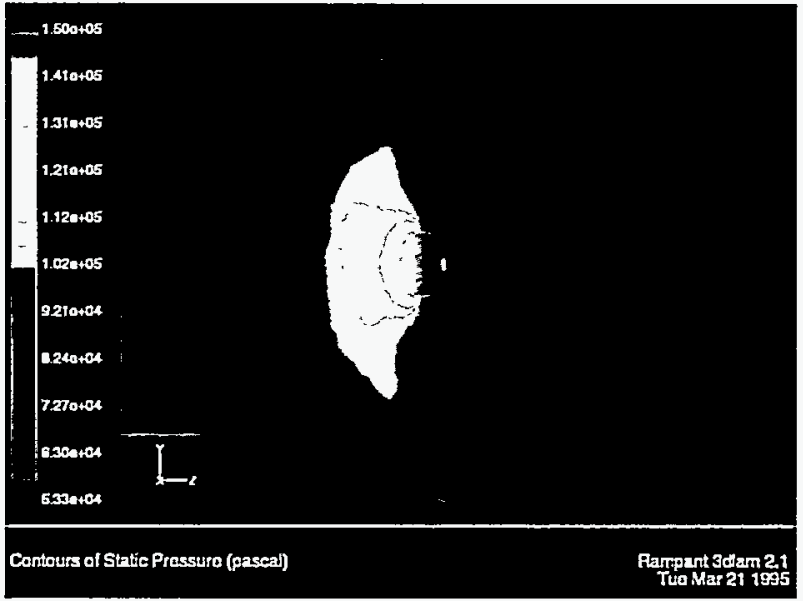

Figure 13. Centerline plane-slice of static pressure. (Ribbon canopy, Mach 0.75 flowfield.) 


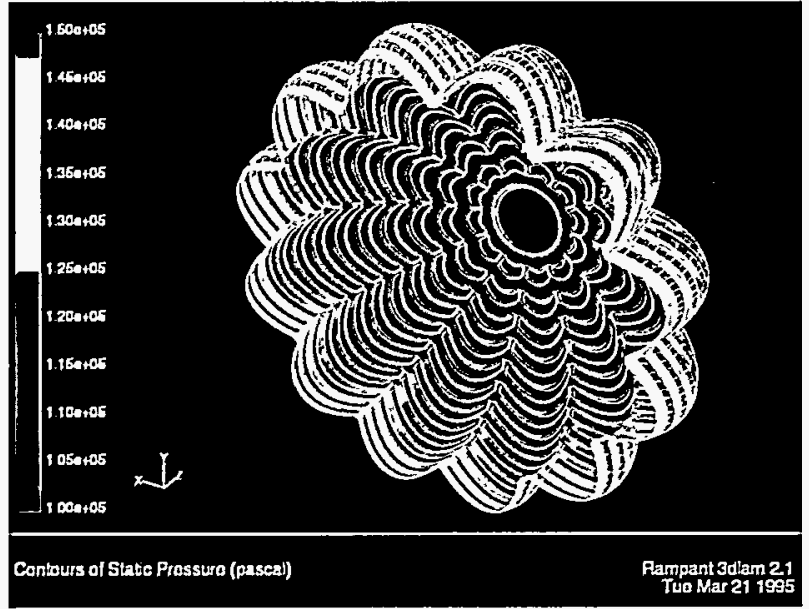

Figure 14. Canopy internal surface pressure distribution. ( Ribbon canopy, Mach 0.75 flowfield.)

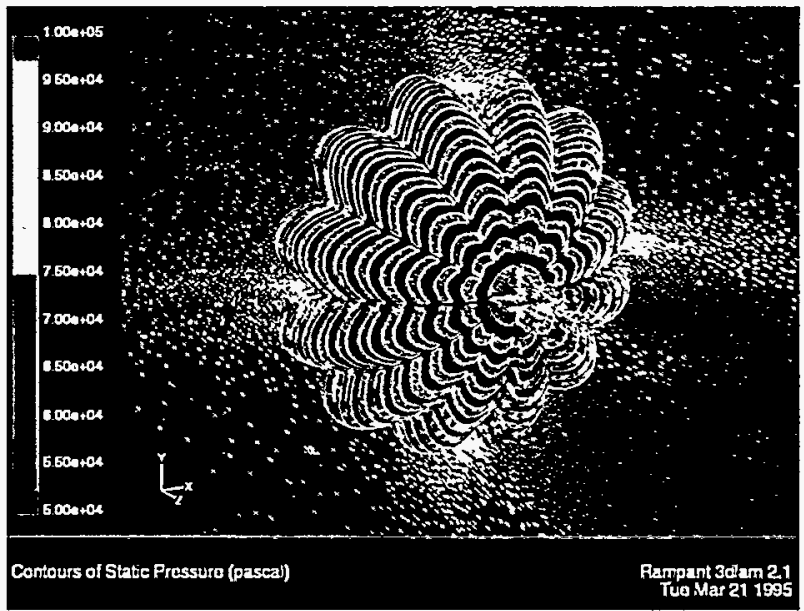

Figure 15. Canopy external surface pressure distribution. ( Ribbon canopy, Mach 0.75 flowfield.)

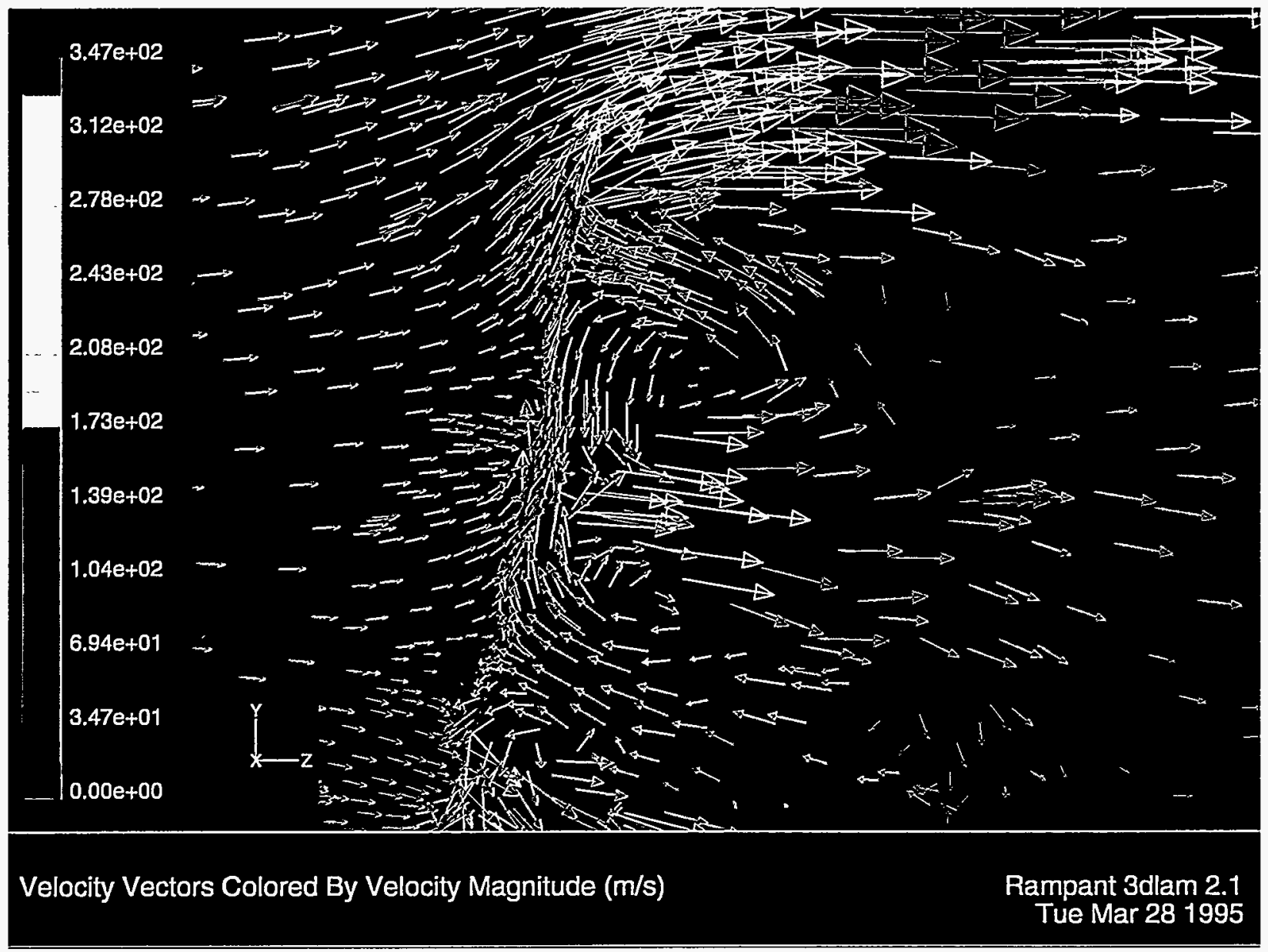

Figure 16. Centerline plane-slice of velocity vectors exhibiting flow through the gap and vent and the ribbon vortex. (Ribbon canopy, Mach 0.75 flowfield.) 


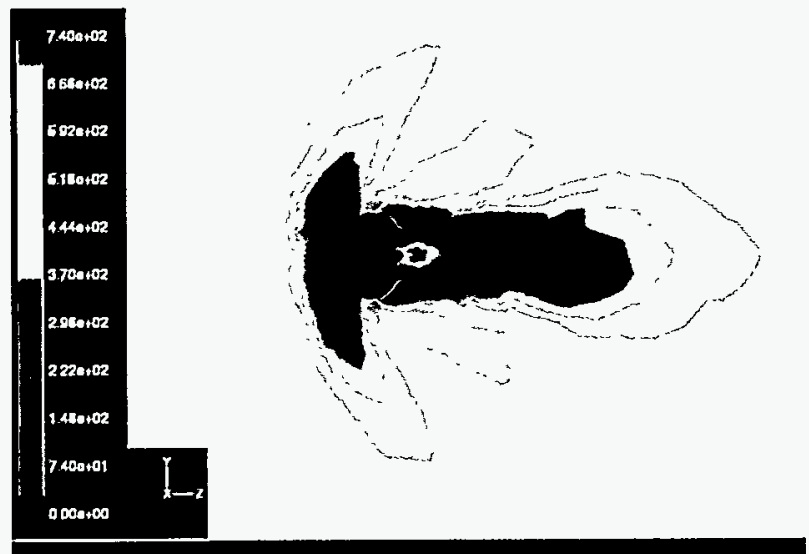

Contours of Volocily Magritude (m/s)

Pampant 3dtam 21
Mon Mar 201895

Figure 17. Centerline plane-slice of velocity magnitude. ( Solid canopy, Mach 1.5 flowfield.)

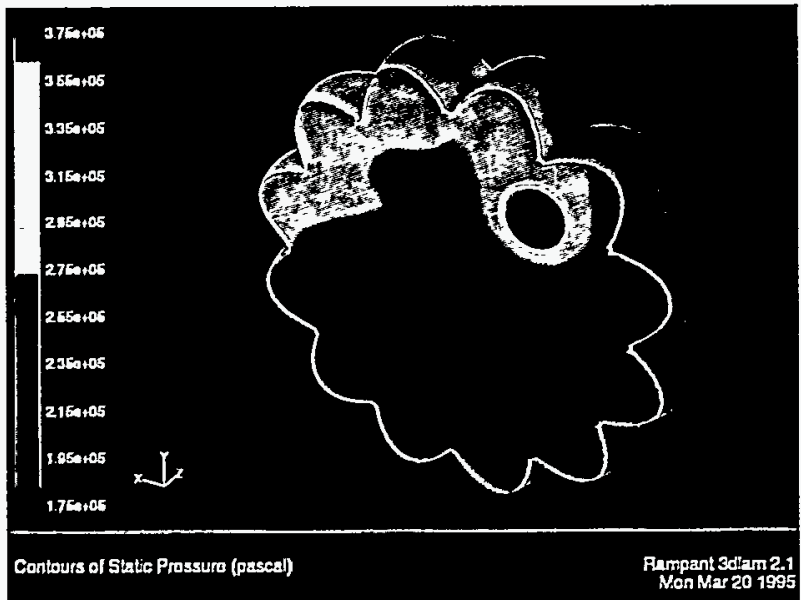

Figure 19. Canopy internal surface pressure distribution. ( Solid canopy, Mach 1.5 flowfield.)

Finally, the wake region extends further downstream than the corresponding subsonic case, perhaps shielded from integrating into the supersonic freestream by the Mach cone.

As displayed in Figure 21, the two counter-rotating toroidal vortices are present in the wake region. However, there are slight differences between this supersonic case and the subsonic case displayed in Figure 11. In the supersonic case, the secondary vortex is larger, the centroid of the primary vortex is nearer the canopy and the stagnation arcs have moved closer together.

Neglecting the skirt and vent band edge effects, the internal canopy pressure distribution is essentially uniform, with less than an $8 \%$ spatial variation. The external canopy pressure distribution is axisymmetric, with the majority of the surface experiencing $36 \mathrm{kPa}$ pressure, except for the locally higher pressure at the skirt and vent band

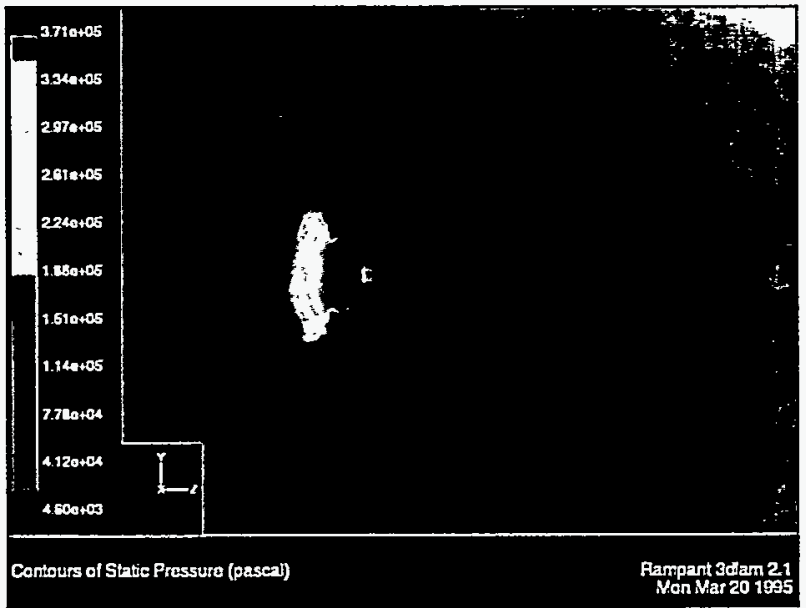

Figure 18. Centerline plane-slice of static pressure. ( Solid canopy, Mach 1.5 flowfield.)

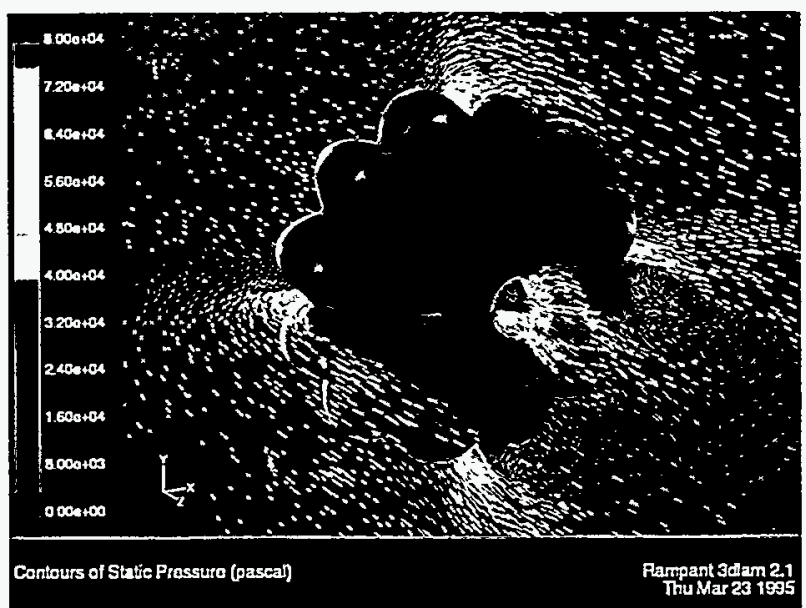

Figure 20. Canopy external surface pressure distribution. ( Solid canopy, Mach 1.5 flowfield.)

and the extremely low $4 \mathrm{kPa}$ pressure registered at a region just forward of the maximum inflated diameter. The canopy differential surface pressure results range from 320 to $340 \mathrm{kPa}$ over most of the canopy, with $370 \mathrm{kPa}$ just forward of the maximum inflated diameter and 100 $\mathrm{kPa}$ experienced at the vent and skirt band.

In an effort to assess the accuracy of the calculated internal canopy pressure distribution, the possible pressure magnitude was bounded. Certainly, the upper bound would be the isentropic freestream stagnation pressure (discounting the potential for added mass effects due to the imposed initial condition), which would not consider the effects of the bow shock. The lower bound would be the stagnation pressure on the backside of a normal shock. This range is $346 \mathrm{kPa}$ to $372 \mathrm{kPa}$. The calculated maximum internal canopy pressure is $371 \mathrm{kPa}$, which is within the established bounds, however, it is intuitively 


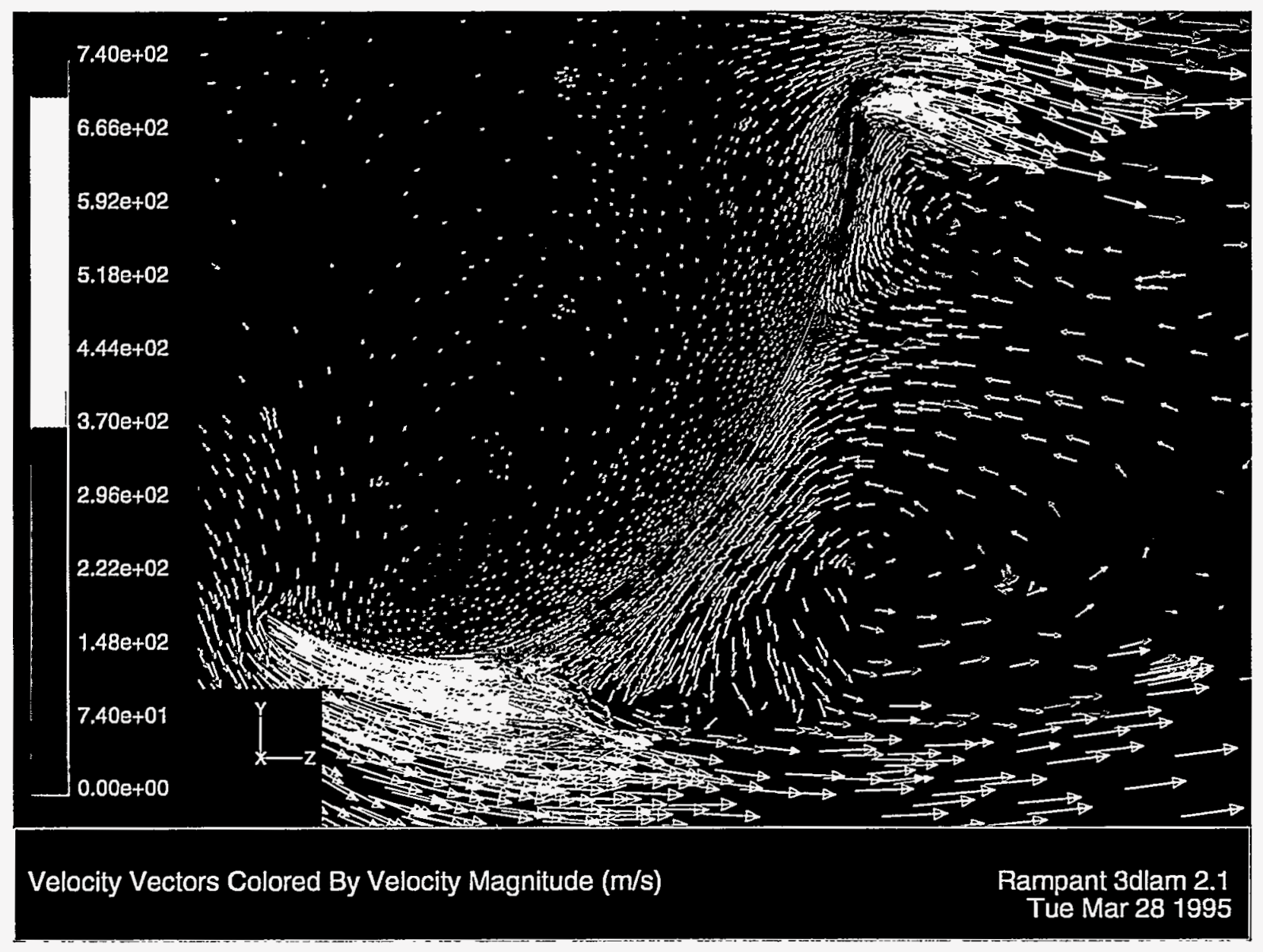

Figure 21. Centerline plane-slice of velocity vectors exhibiting the toroidal vortices.

( Solid canopy, Mach 1.5 flowfield.)

high. Two possible explanations are the deficiency in properly capturing the bow shock physics and the implementation of the "impulsively started" initial condition with it's inherent added mass effects.

\section{Results of the Supersonic, Ribbon Canopy Analysis}

The results of the CFD analysis for the ribbon canopy in the Mach 1.5 flowfield are presented in Figures 22-26. The formation of the bow shock is evident, although the definition is considerably weaker and the placement is noticeably closer to the ribbon canopy than the corresponding solid canopy case. Other salient features include the significant supersonic expansion through the vent and the gaps between the first five ribbons surrounding the vent region. Undoubtedly, the supersonic expansion contributes to the relatively weak wake region which is comprised of primarily high transonic and supersonic flow.

Neglecting ribbon edge effects, the internal and external canopy pressure distributions are substantially uniform, with the notable exception being the skirt region. The in- ternal canopy pressure distribution decreases monotonically approximately $18 \%$ from the uniform pressure over the last four ribbons, while the external distribution increases monotonically by a factor of 30 from the uniform pressure over the last six ribbons. The canopy differential surface pressure distribution ranges from $400 \mathrm{kPa}$ at the vent to $170 \mathrm{kPa}$ at the skirt, with the edge of the ribbons experiencing a relatively low differential pressure of $45 \mathrm{kPa}$.

Considering the accuracy of the computed intemal canopy pressure distribution, the previous pressure bound ( 346 to $372 \mathrm{kPa}$ ) applies to the current ribbon canopy case. The observation of a weaker definition bow shock should contribute to a higher stagnation pressure behind the bow shock (i.e., the lower bound being increased). Indeed, the maximum computed internal canopy pressure is shown to be $400 \mathrm{kPa}$, which exceeds the upper bound by $7.5 \%$. The cause of the high internal canopy pressure is attributed to the "impulsively started" initial condition and the reported "semi-converged" solution. 


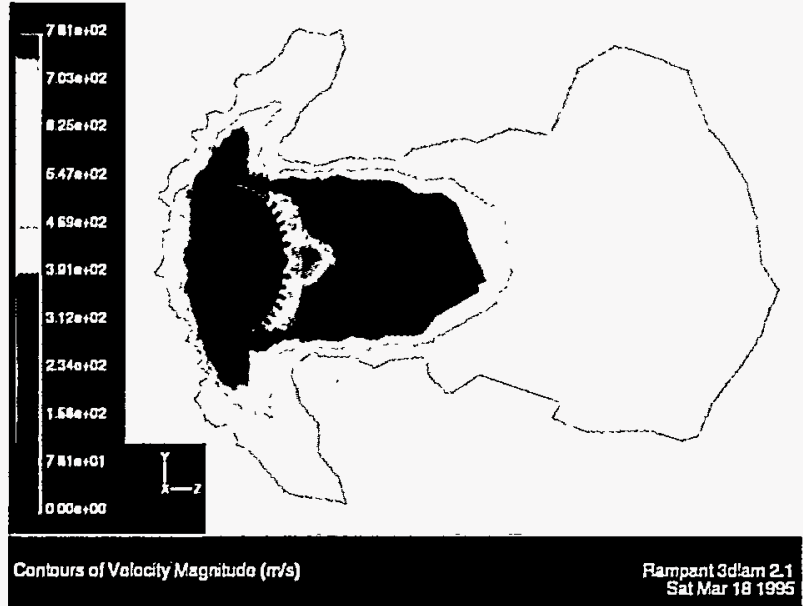

Figure 22. Centerline plane-slice of velocity magnitude. ( Ribbon canopy, Mach 1.5 flowfield.)

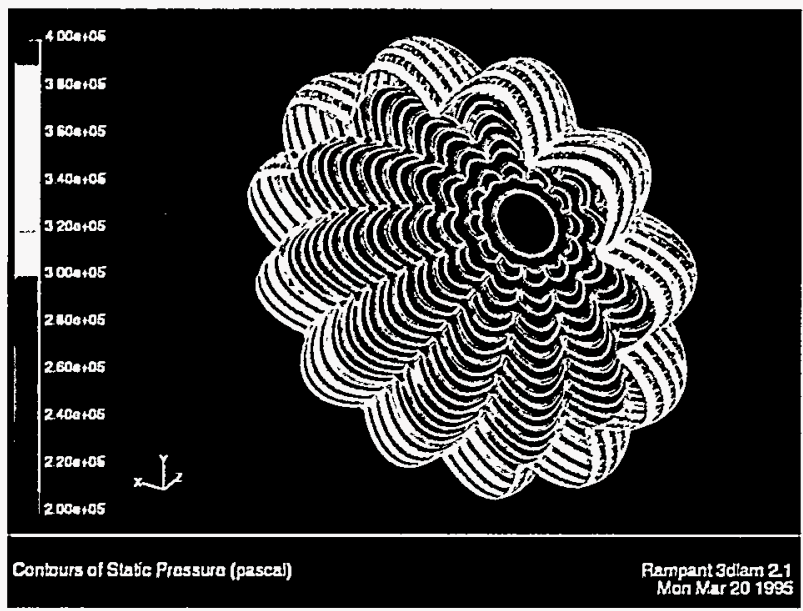

Figure 24. Canopy internal surface pressure distribution. ( Ribbon canopy, Mach 1.5 flowfield.)

\section{Conclusions}

Computational fluid dynamic studies of solid and ribbon 12-gore parachute canopies in subsonic and supersonic flow have been presented. Results included characterization of the local and global flowfield and the internal and external canopy surface pressure distributions.

Generally, the following observations are valid, with the exceptions noted. The internal canopy surface pressure distributions were substantially uniform, with the largest variation being $18 \%$ for the supersonic, ribbon canopy case. Typically, the maximum pressure was recorded in the vent region, with a monotonic decrease in pressure noted from the maximum inflated diameter to the skirt.

The external canopy surface pressure distribution demonstrated significantly more variation. Generally, the ribbon canopies were much more uniform than their solid canopy counterparts, demonstrating a monotonic pres-

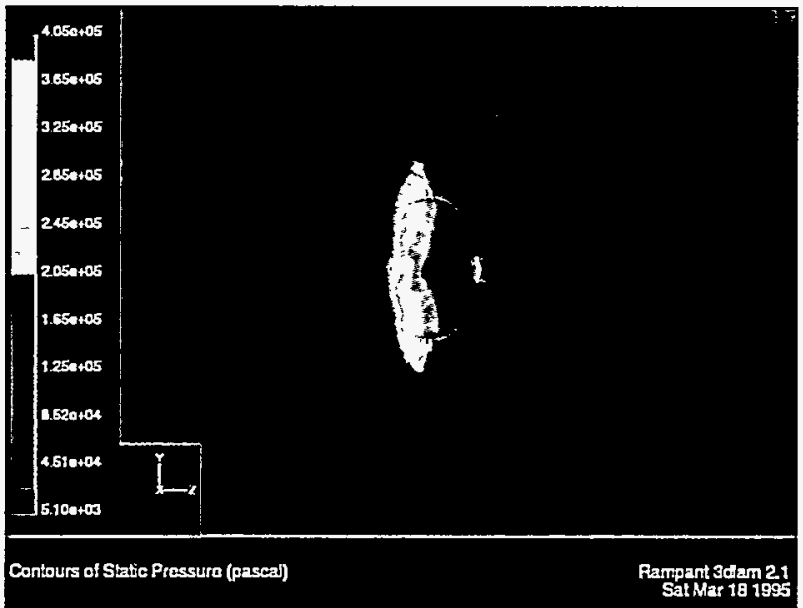

Figure 23. Centerline plane-slice of static pressure. ( Ribbon canopy, Mach 1.5 flowfield.)

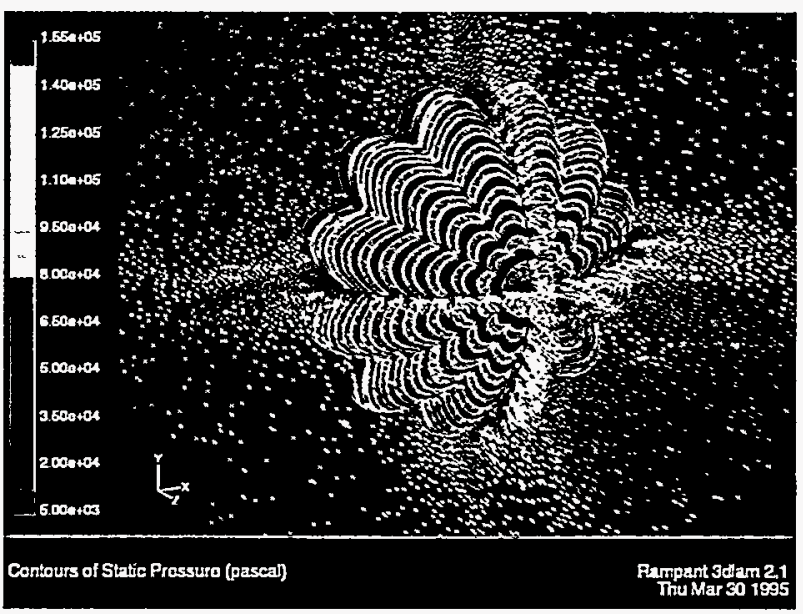

Figure 25. Canopy external surface pressure distribution. (Ribbon canopy, Mach 1.5 flowfield.)

sure increase from the maximum inflated diameter to the skirt. The solid canopies exhibited pressure distributions which were characterized as axisymmetric and nonmonotonic. In particular, the subsonic case demonstrated a high degree of non-uniformity.

The flowfield features are both expected and interesting. The simulations captured the pressure disturbance which propagated upstream of the bluff body canopy, including the ill-defined bow shock for the supersonic cases. The flow accelerated supersonically around the skirt and through the vent for all the cases, and through the gaps of the ribbon parachute canopy. The computed wake region, although present, was noted to be intuitively too small. Two counter-rotating toroidal vortices developed in the near-wake region of the solid canopies, while small vortices were formed behind the ribbons in the vent region of the ribbon canopies. 